\title{
El control interno en las PYMES: Caso Empresa Noble L.G.A.
}

\author{
Internal Control In SMEs: Case of the Noble Company L.G.A. \\ Pedro Enrique Díaz Córdova. ${ }^{1}$, Lucia Margoth Moreno Tapia. ${ }^{2}$, Auria Germania \\ Olvera Anchundia. ${ }^{3} \&$ Karen Liseth Osorio Muñoz. ${ }^{4}$
}

DOI: https://doi.org/10.33262/visionariodigital.v5i2.1641

\begin{abstract}
.
Internal control is of vital importance in the business field given its positive impact that it can generate within an organization, this research will aim to evaluate internal control in achieving the objectives of the company "Ferretería Noble L.G.A;" of the La Maná canton classified within SMEs, the methodology used was based on a qualitative study and a descriptive and bibliographic design, the population was made up of all staff, The company is under the family management and the results obtained determine that they do not have of a formal organizational structure, as well as the absence of a manual of functions, policies and procedures, which allow the fields of action of the collaborators to be delimited and which contribute to the orderly integration of activities and standards to achieve business goals and objectives, in addition to a high component risk level and a low confidence level. It is concluded that it is important to have an adequate internal control system, it depends on avoiding or reducing risks and fraud within the organization and that its implementation is the responsibility of the administration and that it must be applied without considering the size, activity, assets or structure of the same that allows to achieve reasonable security in financial information, decision-making, the development of its activities, and compliance with the legal regulations in force in the country.
\end{abstract}

1 Universidad Técnica de Cotopaxi, Carrera de Contabilidad y Auditoría. La Maná, Ecuador, pedro.diaz0606@utc.edu. https://orcid.org/0000-0003-3416-5033

2 Universidad Técnica de Cotopaxi, Carrera de Contabilidad y Auditoría. La Maná, Ecuador, lucia.moreno6613@utc.edu.ec.

3 Universidad Técnica de Cotopaxi, Carrera de Contabilidad y Auditoría. La Maná, Ecuador, auria.olvera8422@utc.edu.

4 Universidad Técnica de Cotopaxi, Carrera de Contabilidad y Auditoría. La Maná, Ecuador, karen.osorio3628@utc.edu. 
Keywords: SMEs, internal control, fraud, risk.

\section{Resumen.}

El control interno es de vital importancia en el ámbito empresarial dado su impacto positivo que este puede generar dentro de una organización, la presente investigación tendrá como fin evaluar el control interno en el logro de los objetivos de la empresa "Ferretería Noble L.G.A;" del cantón La Maná clasificada dentro de las PYMES, la metodología utilizada se basó en un estudio cualitativo y de diseño descriptivo y bibliográfico, la población lo constituyo todo el personal, La empresa se encuentra bajo la dirección familiar y los resultados obtenidos determino que no disponen de una estructura organizativa formal, así como la ausencia de un manual de funciones, políticas y procedimiento, que permitan delimitar los campos de acción de los colaboradores y que contribuyan a integrar de forma ordenada las actividades y normas para alcanzar las metas y objetivos empresariales, además de un nivel de riesgo por componentes alto y un nivel de confianza bajo. Se concluye que es importante contar con un adecuado sistema de control interno el mismo, de él depende evitar o disminuir riesgos y fraudes dentro de la organización y que su implementación es responsabilidad de la administración y que debe ser aplicado sin considerar el tamaño, la actividad, activos o estructura de la misma que permita alcanzar una seguridad razonable en la información financiera, la toma de decisiones, el desarrollo de sus actividades, y cumplimiento de la normativa legal vigente en el país.

Palabras claves: PYMES, control interno, fraude, riesgo.

\section{Introducción.}

Ante el acentuado proceso de globalización, el creciente y continuo desarrollo de los mercados y un vertiginoso avance tecnológico con los correspondientes riesgos que esto implica, inducen a las empresas a disponer de información cada vez más confiable, oportuna y especializada que mitigue los escándalos financieros y contables dentro de las organizaciones. El presente trabajo de investigación busca establecer la importancia del control interno en las pequeñas empresas y su utilidad como medio para la optimización de los recursos, mejorar el nivel de productividad y la sostenibilidad en el mercado.

(Catágora, 2013) Las crisis financieras, quiebras emblemáticas de reconocidas empresas comerciales, fraudes ineludibles, estados financieros manipulados; en el plano internacional y nacional han demostrado en la historia que una de sus causas fue la ausencia e insuficiente funcionamiento de los controles internos idóneos sobre las operaciones e información financiera que debieron asegurar el correcto uso de los recursos aportados por los dueños en el sector privado y por los ciudadanos en el caso del sector público. 
El control interno es todo un sistema de controles integrales utilizados por las empresas y además lo establece la dirección o gerencia para que estas puedan realizar sus procesos administrativos de manera secuencial y ordenada, con el fin de proteger sus activos, salvaguardarlos y asegurarlos en la medida posible, la exactitud y la veracidad de sus registros contables; sirviendo a su vez de marco de referencia o patrón de comportamiento para que las operaciones y actividades en las diferentes áreas de las empresas fluyan con mayor facilidad. (Palacios, 2003)

Una pyme se crea con un mínimo de recursos y el mayor o menor conocimiento respecto a una actividad o un mercado, que en algunas ocasiones resultan "suficientes" para el emprendimiento, pero no para que la empresa se mantenga y se convierta en una empresa innovadora y competitiva. La dificultad para conseguir fuentes de financiamiento se deriva regularmente de la poca claridad que tienen sobre los objetivos y las metas que se desean alcanzar, y de la ausencia de planes estratégicos que generen un escenario ordenado y acorde con el plan de negocio. (Puyana \& Matallana, 2002)

Así mismo, cabe resaltar que la empresa que aplique controles internos en sus operaciones, podrá conocer con seguridad la situación real de las mismas, debido a la importancia de tener una planificación que sea capaz de verificar que los controles se cumplan para darle una mejor visión sobre su gestión, además es importante tener un buen sistema de control interno en las empresas debido a lo práctico que resulta al medir la eficiencia y la productividad al momento de implantarlos; en especial si se centra en las actividades básicas que ellas realizan, pues de ello dependen para mantenerse en el mercado. (Chacón, 2020)

Desde la perspectiva citada anteriormente podemos mencionar que bajo este contexto es necesario que las empresas canalicen sus recursos humanos, materiales, tecnológicos y financieros en aras de alcanzar las metas y objetivos propuestos por la administración considerando al control interno como herramienta fundamental de una empresa para la sostenibilidad y crecimiento de la misma, si bien la implementación de un control interno no garantiza por si solo el éxito empresarial, un adecuado control interno contribuye a disminuir los riesgos de fraude dentro de una organización independientemente de su tamaño y estructura. Se realizó un estudio cualitativo, bibliográfico y documental.

En el presente trabajo de investigación se evaluó el control interno de la empresa “Ferretería Noble L.G.A.” Del cantón La Maná. Con el fin de establecer como se está llevando los procesos dentro de la organización para que la información recabada permita mejorar el desempeño de las actividades, estableciendo para ello políticas y procedimientos adecuados para el logro de sus objetivos y metas, por lo cual se considera pertinente la presente investigación. 


\section{Desarrollo}

Al evaluar el control interno, el auditor obtiene información detallada sobre los posibles riesgos que pueden afectar negativamente a la organización, así como excepciones de control interno, deficiencias e inconsistencias y algunas veces, indicios de irregularidades y fraudes (Díaz de Santos, 2006).

\section{Definición del control interno basado en el Coso}

Control interno es un proceso, ejecutado por la junta directiva o consejo de administración de una entidad, por su grupo directivo (gerencial) y por el resto del personal, diseñado específicamente para proporcionar seguridad razonable de conseguir en la empresa las tres siguientes categorías de objetivos:

- $\quad$ Efectividad y eficiencia de las operaciones.

- Suficiencia y confiablidad de la información financieras.

- $\quad$ Cumplimento de las leyes y regulaciones aplicables. (Estupiñan R. , 2014)

\section{Componentes del control interno}

Los componentes del control interno están relacionados entre sí, que tienen su relación de como la administración dirige la empresa, de la misma forma están vinculados al proceso administrativo, es así que tenemos la siguiente clasificación:
a) Ambiente de control
b) Evaluación de riesgos
c) Actividades de control
d) Información y comunicación y,
e) Supervisión y seguimiento

Se debe tener presente que el control interno no son pasos secuenciales que siguen un orden determinado y que el un componente incide en el siguiente sino más bien es un proceso multidireccional El control interno, no consiste en un proceso secuencial, en donde alguno de los componentes afecta solo al siguiente, sino en un proceso multidireccional constante y repetitivo donde cada uno de sus componentes tiene incidencia en más de un componente logrando de esta manera un sistema integrado que reacciona activamente a las diferentes condiciones que se presentan. De esta manera, el control interno difiere por ente y tamaño y por sus culturas y filosofías de administración. Así mientras todas las entidades necesitan de cada uno de los componentes para mantener el control sobre sus actividades, el sistema de control interno de una entidad generalmente se percibirá muy diferente al de otra. (Estupiñan R. , 2014) 


\section{Componentes del control interno basado en el COSO I.}

A continuación, se detalla cada uno de los cinco componentes del control interno (Coso I):

\section{Ambiente de control}

(Estupiñan R. , 2014) manifiesta que este componente establece un entorno un entorno que estimule e influencie la actividad del personal de la empresa en relación al control de sus actividades. Sobre este primer componente se sustenta los otros componentes fundamentales para la realización de los objetivos de control. El componente de ambiente de control es el pilar fundamental de los otros cuatro componentes de control a suministrar una diciplina y una estructura para el control, así como incidir en la manera como: Se estructuran las actividades del negocio, se asigna autoridad y responsabilidad, se organiza y desarrolla la gente, se comparten y comunican los valores y creencias y el personal toma conciencia de la importancia del control.

Rivas (2011) distingue los siguientes factores a considerar en el primer componente de ambiente de control: (1) Integridad y valores éticos; (2) Compromiso por la competencia; (3) Consejo de directores o comité de auditoría; (4) Filosofía y estilo operativo de la gerencia; (5) Estructura organizacional; (6) Asignación de autoridad y responsabilidades y; (7) Políticas y procedimientos de recursos humanos. (115-136)

El componente de ambiente de control tiene una gran incidencia de cómo se llevan a cabo las operaciones, se fijan los objetivos y se minimiza el riesgo, de igual manera tiene una relación con el comportamiento de los sistemas de información y la supervisión de forma general. A su vez es influenciado por la historia de la entidad y su nivel de cultura administrativa.

\section{Evaluación de riesgos}

El componente de evaluación de riesgos es el proceso que busca la identificación y análisis de los riesgos más sobresalientes y que tienen estrecha relación con el logro de los objetivos para así poder administrar tales riesgos y establecer la forma en que deben ser enfrentados por la empresa y como pueden ser mejorados.

Mantilla y Blanco (2005) manifiesta que la evaluación de riesgos es: "La valoración de riesgos es la identificación y análisis de los riesgos relevantes para la consecución de los objetivos, formando una base para la determinación de cómo deben administrarse los riesgos. Dado que las condiciones económicas, industriales, reguladoras y de operación continuarán cambiando, se necesitan mecanismos para identificar y tratar los riesgos especiales asociados con el cambio" (p.39) 
Por otro lado, Rivas (2011) indica que: Todas las empresas, independientemente de su tamaño, estructura, naturaleza o clase de industria, enfrentan riesgos en todos los niveles. Los riesgos afectan la destreza de la entidad para sobrevivir. Por lo que, la identificación de los objetivos es una condición previa para la valoración de riesgos. Primero, deben definirse los objetivos a fin de que la administración pueda identificar los riesgos y determinar las acciones necesarias para administrar. La definición de objetivos es una parte clave del proceso administrativo por ser requisito previo para un control interno eficaz. Así mismo, se refiere a los mecanismos necesarios para identificar y manejar riesgos específicos asociados con los cambios, tanto los que influyen en el entorno de la organización como en el interior de la misma.

\section{Actividades de control}

Las actividades de control son aquellas normas, políticas y procedimientos que establece la gerencia y que debe ser llevado a cabo para poder cumplir diariamente con las actividades asignadas, enfocadas a controlar los riesgos.

También son políticas y procedimientos que se desarrollan a través de toda la organización y garantizan que las directrices de la gerencia se llevan a cabo y los riesgos se administren de manera que se cumplan los objetivos.

De acuerdo a Estupiñán (2006) las actividades de control son aquellas que realiza la gerencia y demás personal de la organización para cumplir diariamente con las actividades asignadas. Estas actividades están expresadas en las políticas, sistemas y procedimientos. Ejemplos de estas actividades son la aprobación, la autorización, la verificación, la conciliación, la inspección, la revisión de indicadores de rendimiento, la salvaguarda de los recursos, la segregación de funciones, la supervisión y entrenamiento adecuados. Las actividades de control tienen distintas características. Pueden ser manuales o computarizadas, administrativas u operacionales, generales o específicas, preventivas o detectivas. Sin embargo, lo trascendente es que, sin importar su categoría o tipo, todas ellas están apuntando hacia los riesgos (reales o potenciales) en beneficio de la organización, su misión y objetivos, así como la protección de los recursos propios o de terceros en su poder. Las actividades de control son importantes no sólo porque en sí mismas implican la forma correcta de hacer las cosas, sino debido a que son el medio idóneo de asegurar en mayor grado el logro de los objetivos (pp. 32-33)

Así también Mantilla (2000) señala los siguientes procedimientos de control: revisiones de alto nivel, funciones directivas o actividades administrativas, procesamiento de información, controles físicos, indicadores de desempeño y segregación de responsabilidad.

\section{Factores de las actividades de control}

- Revisiones de alto nivel y actividades administrativas. 
- $\quad$ Proceso de información.

- $\quad$ Controles físicos.

- $\quad$ Indicadores de desempeño.

- $\quad$ Segregación de responsabilidades.

- Políticas y procedimientos.

\section{Tipos de controles}

- Detectivos: Diseñado para detectar hechos indeseables.

- $\quad$ Preventivos: Diseñado para prevenir resultados indeseables.

- Correctivos: Diseñado para corregir efectos de un hecho indeseable.

\section{Sistemas de información y comunicación.}

Los sistemas de información y comunicación es un aspecto elemental y estratégico que establece un vínculo entre la empresa y su entorno con el fin de facilitar sus operaciones, esta información debe ser recogida de forma oportuna, debe ser relevante y trasmitida de manera adecuada que permite a las personas entender y poder cumplir con cada una de sus responsabilidades y los objetivos establecidos por la organización.

Quinaluisa et al. (2018) manifiesta que son elementos esenciales en una estructura de control interno. La información acerca del ambiente de control, la evaluación de los riesgos, los procedimientos de control y la supervisión, resulta necesaria para que los administradores puedan dirigir las operaciones y garantizar el empleo de las normativas legales, reglamentarias y de información. Este componente se refiere, entonces, a los métodos empleados para identificar, reunir, clasificar, registrar e informar acerca de las operaciones de la entidad y para conservar la contabilidad de los activos relacionados.

En lo referente a los sistemas de información y comunicación Carmona et al (2007) menciona que actualmente no se concibe la gestión de una entidad sin sistemas de información, integrado por el sistema contable, el sistema estadístico, y la información para la gestión, en el caso de esta se incluye entre otras: Planes, informes y análisis de la ejecución de los planes, evaluaciones del cumplimiento de los objetivos, flujo de ideas, intercambio de información, comunicación abierta dentro de la organización, comunicación efectiva con el exterior, análisis de actividades e indicadores, informes de auditorías internas y externas, etcétera. Todo el personal, especialmente el que cumple importantes funciones operativas o financieras, debe recibir y entender el mensaje de la dirección de que las obligaciones en materia de control son esenciales. Así mismo conocer su papel en el sistema de control interno, así como sus actividades individuales se relacionan con el trabajo de los demás (pp. 151-171) 


\section{Supervisión y monitoreo}

La supervisión y monitoreo es un proceso dirigido a evaluar la calidad del control interno, su adecuado funcionamiento a través del tiempo y buscando permanentemente un mejoramiento continuo.

El Committee of Sponsoring Organizations of the Treadway Commission (2013) en referencia a las actividades de supervisión manifiesta que: Las evaluaciones continuas, las evaluaciones independientes o una combinación de ambas se utilizan para determinar si cada uno de los cinco componentes del control interno, incluidos los controles para cumplir los principios de cada componente, están presentes y funcionan adecuadamente. Las evaluaciones continuas, que están integradas en los procesos de negocio en los diferentes niveles de la entidad, suministran información oportuna. Las evaluaciones independientes, que se ejecutan periódicamente, pueden variar en alcance y frecuencia dependiendo de la evaluación de riesgos, la efectividad de las evaluaciones continuas y otras consideraciones de la dirección. Los resultados se evalúan comparándolos con los criterios establecidos por los reguladores, otros organismos reconocidos o la dirección y el consejo de administración, y las deficiencias se comunican a la dirección y al consejo, según corresponda (p. 5)

Por otro lado, Estupiñán (2006) manifiesta que: La gerencia debe llevar a cabo la revisión y evaluación sistemática de los componentes y elementos que forman parte de los sistemas de control. Lo anterior no significa que tengan que revisarse todos los componentes y elementos, como tampoco que deba hacerse al mismo tiempo. Ello dependerá de las condiciones específicas de cada organización, de los distintos niveles de efectividad mostrado por los distintos componentes y elementos de control. La evaluación debe conducir a la identificación de los controles débiles, insuficientes o innecesarios, para promover con el apoyo decidido de la gerencia, su robustecimiento e implantación. Esta evaluación puede llevarse a cabo de tres formas: durante la realización de las actividades diarias en los distintos niveles de la organización; de manera separada por personal que no es el responsable directo de la ejecución de las actividades (incluidas las de control) y mediante la combinación de las dos formas anteriores (p. 37).

\section{Matriz de riesgo del control interno}

Una matriz de riesgos es una sencilla pero eficaz herramienta para identificar los riesgos más significativos inherentes a las actividades de una empresa, tanto de procesos como de fabricación de productos o puesta en marcha de servicios. Por lo tanto, es un instrumento válido para mejorar el control de riesgos y la seguridad de una organización. A través de este instrumento se puede realizar un diagnóstico objetivo y global de empresas de diferentes tamaños y sectores de actividad. Asimismo, mediante la matriz de riesgo es posible evaluar la efectividad de la gestión de los riesgos, tanto financieros como operativos y estratégicos, que están impactando en la misión de una determinada organización. (ISOTools, 2015) 


\section{Pasos para la elaboración de una matriz de riesgo}

Los pasos para la elaboración de una matriz de riesgo son los siguientes:

\section{1) Identificación de riesgos}

El primer paso consiste en la identificación de las actividades principales de una organización y de los riesgos inherentes a estas. De una manera general, se puede entender como riesgos la posibilidad de que a una empresa le sea imposible cumplir con alguno de sus objetivos. Una vez establecidas todas las actividades, ya es posible prever los posibles riesgos y los motivos o factores que intervienen en su manifestación y grado, distinguiéndose en riesgos intrínsecos, que serían aquellos que provienen directamente de la propia empresa, y extrínsecos, factores de incertidumbre provocados por eventos externos o macro económicos que pueden tener un impacto sobre la actividad de nuestra empresa.

\section{2) Evaluar la probabilidad de que se acabe confirmando el riesgo.}

El siguiente paso consistiría en determinar la probabilidad de que, efectivamente, el riesgo ocurra, así como un cálculo de los efectos potenciales del mismo. Se trata, por lo tanto, de una valorización del riesgo, lo cual implica un análisis conjunto e interrelacionado de la probabilidad de ocurrencia y del efecto en los resultados globales de la empresa. Los riesgos se pueden valorar en términos cualitativos o cuantitativos, utilizando normalmente valores numéricos o estadísticos, lo que ayuda a tener una base sólida para que la dirección o responsables de la empresa o negocio puedan tomar las decisiones pertinentes.

\section{3) Representación de la matriz de riesgos}

La verdadera utilidad de la matriz de riesgos radica en que ofrezca la posibilidad de tener una idea general de los riesgos de una empresa y la posibilidad de que ocurran con tan solo echarle un vistazo. Por este motivo, la representación de la matriz debe ser en forma de tablas no demasiado complejas donde aparezcan los riesgos, probabilidad de ocurrencia, gravedad de los mismos y, si se desea, acciones para solucionarlos y mitigarlos. Existen aplicaciones informáticas específicas para facilitar su elaboración. (ISOTools, 2015)

\section{Empresa}

Una empresa es una unidad productiva dedicada y agrupada para desarrollar una actividad económica y tienen ánimo de lucro. En nuestra sociedad, es muy común la constitución continua de empresas. En general, se puede definir como una unidad formada por un grupo de personas, bienes materiales y financieros, con el objetivo de producir algo o 
prestar un servicio que cubra una necesidad y, por el que se obtengan beneficios. (Coloma, Franco, \& Cedeño, 2016)

\section{Clasificación según su tamaño}

Grandes empresas: Aquellas empresas cuyo volumen de negocio haya superado durante el año contable. Además, están obligadas a presentar mensualmente las declaracionesliquidaciones referentes a retenciones, impuesto sobre el valor añadido (IVA), impuestos especiales y primas de seguros.

Pymes: A efectos de la agencia tributaria, se consideran PYMES todas aquellas organizaciones que no son grandes empresas con obligación de declarar mensualmente las retenciones sobre rendimientos del trabajo y actividades económicas.

Producción, transformación y/o prestación de servicios: Se refiere a que la empresa puede realizar una o más de las siguientes actividades:

- Fabricar, elaborar o crear cosas o servicios con valor económico.

- Transformar o cambiar, por ejemplo, una materia prima en un producto terminado.

- Prestar servicios.

Satisfacción de necesidades y deseos: La necesidad humana es el estado en el que se siente la privación de algunos factores básicos (alimento, vestido, abrigo, seguridad, sentido de pertenencia, estimación). (Coloma, Franco, \& Cedeño, 2016)

\section{Clasificación de las PYMES, pequeña y mediana empresa (Según la CAN)}

El Programa Estadístico Comunitario de la CAN, Comunidad Andina de Naciones, adoptado mediante Decisión 488, define los preceptos básicos para elaborar las estadísticas comunitarias de las PYMES. Este sistema estadístico regional establece que las PYMES comprenden a todas las empresas formales legalmente constituidas y/o registradas ante las autoridades competentes, que lleven registros contables y/o aporten a la seguridad social, comprendidas dentro de los umbrales establecidos en el artículo 3 de la Decisión 702.

El artículo 3 de la Decisión 702 de la CAN establece los parámetros de acuerdo a lo señalado a continuación: a) Las empresas comprendidas dentro de los siguientes rangos de personal ocupado y de valor bruto de las ventas anuales: (Cámara de Comercio de Quito, 2017). Ver tabla 1. 
Tabla 1.

Clasificación de las PYMES por Estrato, Ventas y Personal Ocupado.

\begin{tabular}{lcccc}
\hline $\begin{array}{l}\text { Variables } \\
\left({ }^{* *}\right)\end{array}$ & $\begin{array}{c}\text { Estrato } \\
\text { I }\end{array}$ & $\begin{array}{c}\text { Estrato } \\
\text { II }\end{array}$ & $\begin{array}{c}\text { Estrato } \\
\text { III }\end{array}$ & $\begin{array}{c}\text { Estrato } \\
\text { IV }\end{array}$ \\
\hline $\begin{array}{l}\text { Personal } \\
\text { ocupado }\end{array}$ & $1-9$ & $10-49$ & $50-$ & $100-$ \\
& & & $99+$ & 199 \\
\hline $\begin{array}{l}\text { Valor } \\
\text { bruto de }\end{array}$ & $\leq 100.0$ & 100.000 & 1.000 .0 & 2.000 .0 \\
$\begin{array}{l}\text { las ventas } \\
\text { anuales }\end{array}$ & 00 & 1.000 .0 & 01 & 01 \\
(US $\$)^{*}$ & & 00 & 2.000 .0 & $\begin{array}{c}5.000 .0 \\
\text { (n) }\end{array}$ \\
\hline
\end{tabular}

Nota: $\left({ }^{*}\right)$ Margen comercial para las empresas comerciales $\left({ }^{* *}\right)$ Prevalecerá el valor bruto de las ventas anuales sobre el criterio del personal ocupado. Adaptado de clasificación de las empresas. Fuente: Cámara de Comercio de Quito (2017).

\section{Clasificación nacional}

De acuerdo a la clasificación de la CAN, la empresa ferretera Noble L.G.A. estaría clasificada como una micro empresa por el número de personal ocupado.

"La Superintendencia de Compañías, Valores y Seguros, mediante resolución, acogió la clasificación de pequeñas y medianas empresas, PYMES, de acuerdo a la normativa implantada por la Comunidad Andina en su Resolución 1260 y la legislación interna vigente" (Cámara de Comercio de Quito, 2017). Ver tabla 2.

\section{Tabla 2.}

Clasificación de las PYMES por Rango de Ventas y Personal Ocupado.

\begin{tabular}{ccccc}
\hline Variables & Micro & $\begin{array}{c}\text { Pequeña } \\
\text { Empresa }\end{array}$ & $\begin{array}{c}\text { Mediana } \\
\text { Empresa }\end{array}$ & $\begin{array}{c}\text { Grandes } \\
\text { Empresas }\end{array}$ \\
\hline $\begin{array}{c}\text { Personal } \\
\text { ocupado }\end{array}$ & $1-9$ & $10-49$ & $50-199$ & $\geq 200$ \\
\hline $\begin{array}{c}\text { Valor } \\
\text { bruto de } \\
\text { las ventas } \\
\text { anuales }\end{array}$ & $\leq 100.000$ & $\begin{array}{c}100.001 \\
1.000 .000\end{array}$ & 5.000 .000 & $>5.000 .000$ \\
\hline
\end{tabular}


Vol. 5, $\mathrm{N}^{\circ}$ 2, p. 89-105, abril-junio, 2021

\begin{tabular}{ccccc}
\hline & & De US\$ & De US\$ & \\
Monto de & Hasta & 100.001 & 750.001 & \\
activos & US\$ & hasta & hasta & $\geq 4.000 .000$ \\
& 100.000 & US\$ & US\$ & \\
& & 750.000 & 3.999 .999 &
\end{tabular}

Nota: Clasificación Nacional de las PYMES considerando: Personal ocupado, valor bruto de ventas o monto de activos. Adaptado de clasificación de las empresas. Fuente: Cámara de Comercio de Quito (2017).

\section{Metodologia.}

Conforme al objetivo planteado se establecieron diversas actividades metodológicas a fin de obtener información relevante de la situación real de la empresa, para lo cual se determinó una población compuesta por 7 personas, con un nivel de estudios de primaria, secundaria, y universitaria.

La investigación se fundamentó en un estudio cualitativo, bibliográfico y descriptivo, para la recolección de datos se aplicó una entrevista estructurada y cuestionarios a fin de identificar la importancia del control interno dentro de la organización, preguntas que fueron apoyadas en la observación directa no participante que combinadas permitieron enfocarse en los temas más relevantes, que posteriormente fueron clasificadas y analizadas y cuya aplicación está enfocada a los objetivos planteados y que los datos obtenidos evidencien la situación de la empresa "Ferretería Noble L.G.A.".

\section{Resultados}

Uno de los principales problemas que se pudo identificar es que no se encuentra definido de forma adecuada las políticas, principios, procedimientos y mecanismos de evaluación y verificación por parte de la administración. Ver figura 1.

\section{Figura 1.}

Disponen de un sistema o manual de control interno

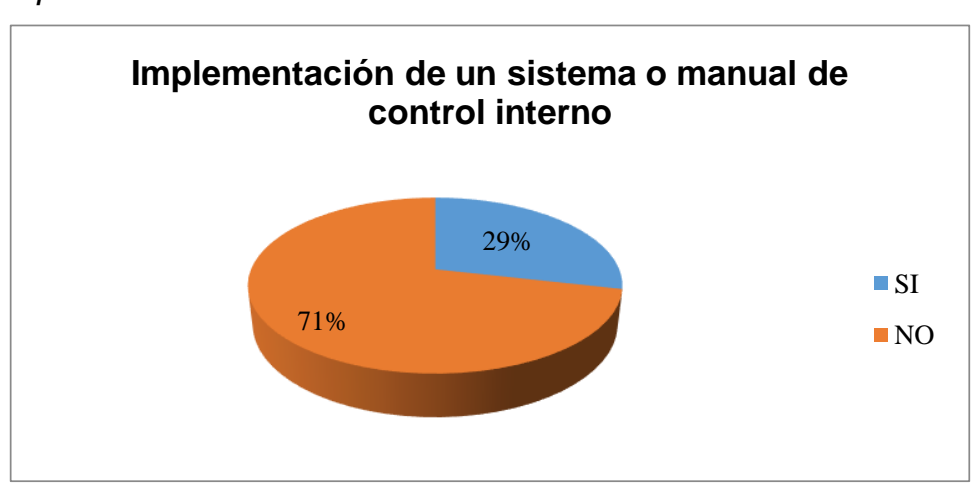

Nota: Datos obtenidos de encuesta a "Ferretería Noble L.G.A.".

Elaboración propia. 
La información contable no es procesada dentro de los tiempos definidos por la administración o entes de control lo que conlleva al incumplimiento de la normativa legal vigente y a la generación de costos por multas e intereses a la empresa. Ver figura 2.

\section{Figura 2.}

Información contable actualizada.

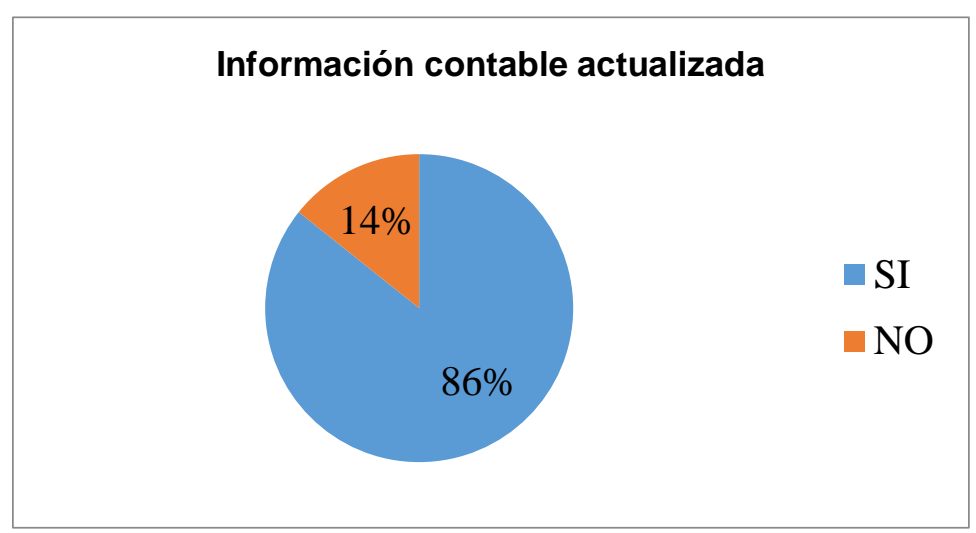

Nota: Datos obtenidos de encuesta a "Ferretería Noble L.G.A.".

Elaboración propia.

Se pudo evidenciar debilidad en la actualización de los inventarios al no existir un control adecuado de la mercadería que permita disponer de registros actualizados y confiables. Ver figura 3.

Figura 3.

Registros de inventarios actualizados

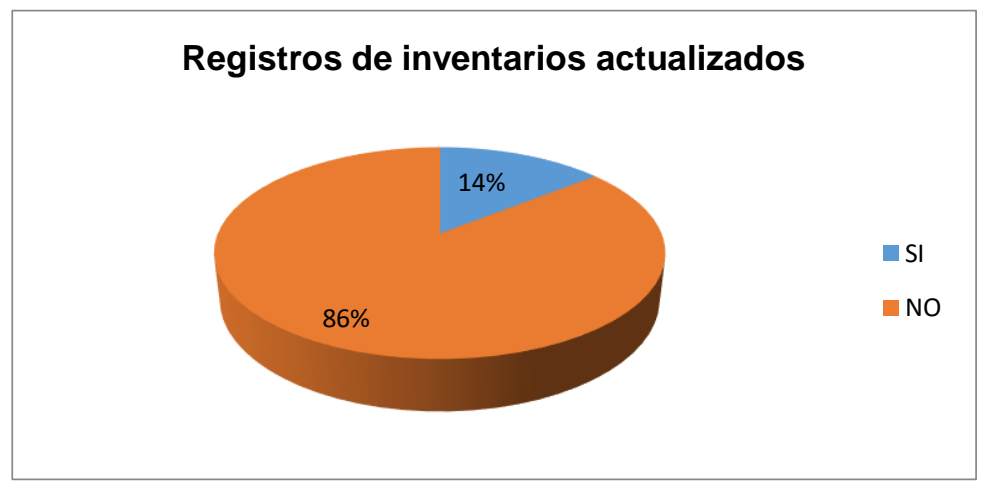

Nota: Datos obtenidos de encuesta a "Ferretería Noble L.G.A.". Elaboración propia.

Por otra parte, del personal que labora en la empresa no cumple con el perfil profesional requerido para el desempeño del cargo acompañado de la falta de capacitación ocasionando dificultad en desempeño de sus funciones. Ver figura 4. 
Figura 4.

Cumplimiento de los empleados con el perfil profesional

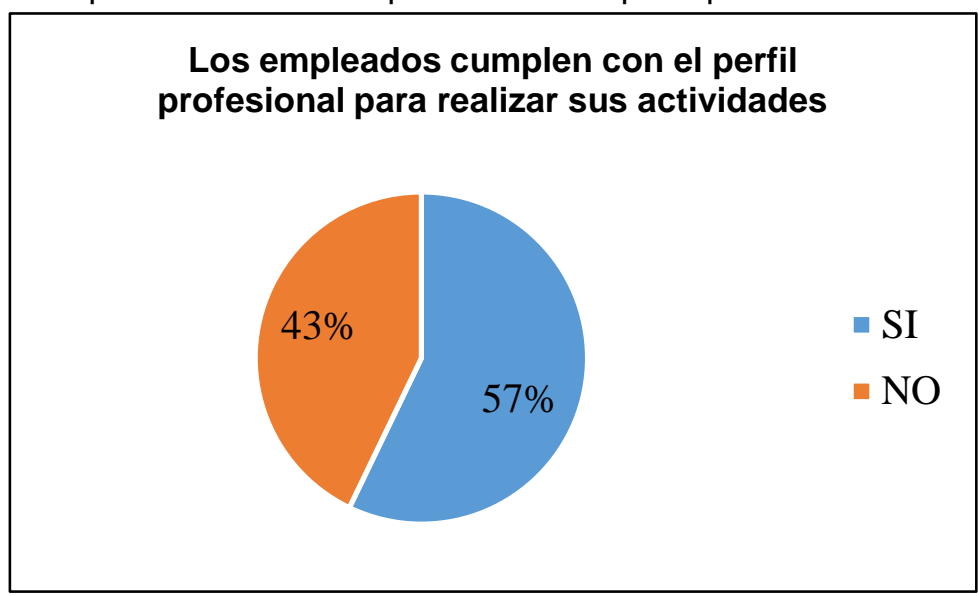

Nota: Datos obtenidos de encuesta a "Ferretería Noble L.G.A.". Elaboración propia.

En relación al análisis total de los componentes en base a los cuestionarios aplicados se llegó a determinar un nivel de confianza bajo 23,81 y un nivel de riesgo alto con el $76,19 \%$, existiendo el menor cumplimiento en el ambiente de control, reflejando la falta de normativa en la organización y una débil estructura organizativa. Ver tabla 3.

\section{Tabla 3.}

Resultados del Cuestionario de Control Interno por Componentes.

\begin{tabular}{lccc}
\hline \multicolumn{4}{c}{ "Ferretería Noble L.G.A" } \\
\hline Componentes & 24 & $8,33 \%$ & $91,67 \%$ \\
\cline { 2 - 4 } No Preqúnta $^{\text {Ambiente de control }}$ & $\begin{array}{c}\text { Nivel de } \\
\text { Confianza }\end{array}$ & $\begin{array}{c}\text { Nivel de } \\
\text { Riesgo }\end{array}$ \\
Valoración de riesgo & 12 & $16,67 \%$ & $83 \%$ \\
Actividades de control & 24 & $33,33 \%$ & $66,67 \%$ \\
Información y comunicación & 16 & $18,75 \%$ & $81,25 \%$ \\
Monitoreo & 8 & $62,50 \%$ & $37,50 \%$ \\
\hline \multicolumn{1}{c}{ TOTAL } & $\mathbf{8 4}$ & $\mathbf{2 3 , 8 1 \%}$ & $\mathbf{7 6 , 1 9 \%}$ \\
\hline
\end{tabular}

Nota: Datos obtenidos de la evaluación al control interno de la empresa " Ferretería Noble L.G.A.". Elaboración propia.

En la parte gerencial existe desconocimiento del proceso administrativo lo que dificulta una buena administración en el manejo del recurso humano y de los procesos administrativos, problemas que se derivan de la falta de capacitación en el área gerencial. 


\section{Conclusiones.}

- Generalmente en las micro, pequeñas y medianas empresas, no se encuentra definido un sistema de administración, razón por lo que los objetivos, metas y planes no se encuentran plasmados en un documento, generando un crecimiento muchas veces descontrolado, con inversiones sin un adecuado análisis, creación de departamentos sin una estructura organizacional, ocasionando pérdidas y fraudes frecuentes, afectando el patrimonio y llevando a un incumplimiento de las leyes y reglamentos que pueden derivarse en un pasivo contingente y que puede ser motivo de iliquidez en la empresa y sostenibilidad de la misma, situación que podría ser prevenida disminuyendo el riesgo y fraude a través de la implementación de un adecuado sistema de control interno.

- En el caso investigado se ha podido determinar que el sistema de control interno es débil, se evidencio falencias en la definición de políticas y procedimientos, así como la falta de una estructura organizacional y definición de funciones de los colaboradores, presentando un nivel de riesgo confianza baja, lo que incide en el normal desenvolvimiento de sus actividades.

- Se debe evitar caer por parte de la administración en la idea que por ser micro empresa no es necesario la implementación de un buen sistema de control interno, toda organización sin considerar su tamaño o actividad debe contar un sistema de control interno adecuado a su tamaño y actividad.

- Finalmente, podemos manifestar que el control interno en las empresas es una tarea ineludible si se quiere lograr mantenerse en un mercado cada vez más competitivo, la implementación de un adecuado sistema de control interno permitirá disminuir errores y fraudes, mejorando los procesos y la presentación de la información financiera fiable que permitirá una mejor toma de decisiones y un crecimiento sostenido.

\section{Referencias Bibliográficas}

Camara de comercio de Quito. (2017). Clasificación de las PYMES, pequeña y mediana empresa. Obtenido de http://www.ccq.ec/wpcontent/uploads/2017/06/Consulta_Societaria_Junio_2017.pdf

Carmona González, M., \& Barrios Hernández, Y. (2007). Nuevo paradigma del control interno y su impacto en la gestión pública. Economía y Desarrollo, 141(1), 152171. Obtenido de http://www.redalyc.org/articulo.oa?id=425541595009

Catágora, F. (2013). Sistemas y procedimientos contables. Venezuela: McGraw Hill.

Chacón, W. (10 de 05 de 2020). aeca. Obtenido de https://www.aeca.es/old/buscador/infoaeca/articulosespecializados/pdf/auditoria/ pdfcontabilidad/15.pdf 
Coloma, Franco, \& Cedeño. (2016). Contabilidad de costos en las empresas. Revista Caribeña de Ciencias Sociales, 3-4.

Committee of Sponsoring Organizations of the Treadway Commission. (2013). Control Interno - Marco Integrado . Obtenido de https://auditoresinternos.es/uploads/media_items/coso-resumenejecutivo.original.pdf

Díaz de Santos, M. (2006). Análisis de Informe COSO I y II. Bogota: Ecoe Ediciones.

Estupiñan, R. (2006). Control interno y fraudes (Segunda ed.). Bogotá: Ecoe Ediciones.

Estupiñan, R. (2014). Control interno y fraudes (Segunda edición ed.). Bogotá, Colombia: Ecoe ediciones.

ISOTools. (06 de 08 de 2015). ISOTools( Plataforma Tecnológica de la Gestión de la Excelencia). Obtenido de https://www.isotools.org/2015/08/06/en-que-consisteuna-matriz-de-riesgos/

Laudon, K., \& Laudon, J. (2012). Sistemas de Información Gerencial (Cuarta ed.). Mexico: Pearson.

Mantilla, S. (2000). Control interno: Estructura conceptual integrada: Herramientas de evaluacion, reporte a partes externas, estructura conceptual y resumen ejecutivo. Colombia: Ecoe ediciones.

Mantilla, S., \& Cante, S. (2005). Auditoría del control interno. Colombia: Ecoe ediciones.

Palacios, Y. (2003). Normas y Procedimientos de la Auditoría Integral. Bogotá: ED.

Puyana, S., \& Matallana, V. (2002). Las Mipymes colombianas y el mercado internacional. Revista Civilizar 2, 67-73.

Quinaluisa Morán, N. V., Ponce Álava, V. A., Muñoz Macías, S. C., Ortega Haro, X. F., \& Pérez Salazar, J. A. (2018). El control interno y sus herramientas de aplicación entre COSO y COCO. Cofin Habana, 12(1), 268-283. Obtenido de http://scielo.sld.cu/scielo.php?script=sci_arttext\&pid=S207360612018000100018

Rivas, G. (2011). Modelos contemporaneos de control interno. Fundamentos teóricos. Observatorio Laboral Revista Venezolana, 4(8), 115-136. Obtenido de http://www.redalyc.org/articulo.oa?id=219022148007

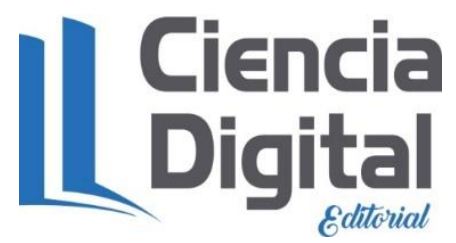




\section{PARA CITAR EL ARTÍCULO INDEXADO.}

Díaz Córdova, P. E., Moreno Tapia, L. M., Olvera Anchundia, A. G., \& Osorio Muñoz, K. L. (2021). El control interno en las PYMES: Caso Empresa Noble L.G.A. Visionario Digital, 5(2), 89-105. https://doi.org/10.33262/visionariodigital.v5i2.1641

\section{DDigital}

El artículo que se publica es de exclusiva responsabilidad de los autores y no necesariamente reflejan el pensamiento de la Revista Visionario Digital.

El artículo queda en propiedad de la revista y, por tanto, su publicación parcial y/o total en otro medio tiene que ser autorizado por el director de la Revista Visionario Digital.
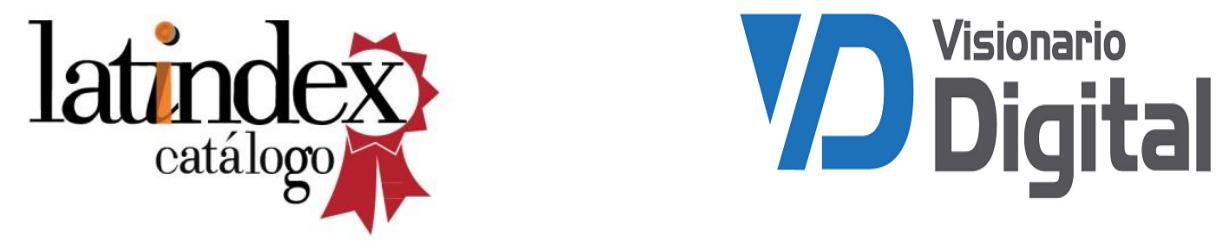